\title{
Cardiopulmonary
}

Support

and Physiology

\section{Myocardial protection with oxygenated esmolol cardioplegia during prolonged normothermic ischemia in the rat}

Ryuzo Bessho, MD, PhD

David J. Chambers, PhD

See related editorial on page 219.
From the Cardiac Surgical Research/Cardiothoracic Surgery, The Rayne Institute, Guy's and St Thomas' NHS Trust, St Thomas' Hospital, London, United Kingdom.

Received for publication June 5, 2001; revisions requested Sept 19, 2001; revisions received Nov 13, 2001; accepted for publication Nov 20, 2001.

Address for reprints: David J. Chambers, $\mathrm{PhD}$, Cardiac Surgical Research/Cardiothoracic Surgery, The Rayne Institute, Guy's and St Thomas' NHS Trust, St Thomas' Hospital, London SE1 7EH, United Kingdom (E-mail: david.chambers@kcl.ac.uk).

J Thorac Cardiovasc Surg 2002;124:340-51

Copyright ( $\odot 2002$ by The American Association for Thoracic Surgery

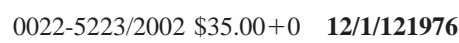

doi: $10.1067 / \mathrm{mtc} .2002 .121976$
Objective: We previously showed that arrest with multidose infusions of high-dose $(1 \mathrm{mmol} / \mathrm{L})$ esmolol (an ultra-short-acting $\beta$-blocker) in oxygenated Krebs-Henseleit buffer (esmolol cardioplegia) provided complete myocardial protection after 40 minutes of normothermic $\left(37^{\circ} \mathrm{C}\right)$ global ischemia in isolated rat hearts. In this study we investigated the importance of oxygenation for protection with esmolol cardioplegia, compared it with that of St Thomas' Hospital cardioplegia, and determined the protective efficacy of multidose esmolol cardioplegia for extended ischemic durations.

Methods: Isolated rat hearts $(n=6$ /group) were perfused in the Langendorff mode at constant pressure $(75 \mathrm{~mm} \mathrm{Hg})$ with oxygenated Krebs-Henseleit bicarbonate buffer at $37^{\circ} \mathrm{C}$. The first part of the first study had four groups: (i) multidose (every 15 minutes) oxygenated (95\% oxygen/5\% carbon dioxide) Krebs-Henseleit buffer during 60 minutes of global ischemia, (ii) multidose deoxygenated (95\% nitrogen $/ 5 \%$ carbon dioxide) Krebs-Henseleit buffer during 60 minutes of global ischemia, (iii) multidose oxygenated esmolol cardioplegia during 60 minutes of global ischemia, and (iv) multidose deoxygenated esmolol cardioplegia during 60 minutes of global ischemia. The second part of the first study had three groups: (v) multidose St Thomas' Hospital solution during 60 minutes of global ischemia, (vi) multidose oxygenated St Thomas' Hospital solution during 60 minutes of global ischemia, and (vii) multidose oxygenated esmolol cardioplegia during 60 minutes of global ischemia. In the second study, hearts were randomly assigned to $60,75,90$, or 120 minutes of global ischemia and at each ischemic duration were subjected to multidose oxygenated constant flow or constant pressure infusion of (i) KrebsHenseleit buffer (constant flow), (ii) Krebs-Henseleit buffer (constant pressure), (iii) esmolol cardioplegia (constant flow), or (iv) esmolol cardioplegia (constant pressure). All hearts were reperfused for 60 minutes, and recovery of function was measured.

Results: Multidose infusion of oxygenated esmolol cardioplegia completely protected the hearts $(97 \% \pm 5 \%)$ after 60 minutes of $37^{\circ} \mathrm{C}$ global ischemia. Deoxygenated esmolol cardioplegia was significantly less protective $(45 \% \pm 8 \%)$. Oxygenation of St Thomas' Hospital solution did not alter its protective efficacy in this study $(70 \% \pm 4 \%$ vs $69 \% \pm 7 \%)$. Infusion of esmolol cardioplegia at constant pressure provided complete protection for 60,75 , and 90 minutes $(104 \% \pm 5 \%$, $95 \% \pm 5 \%$, and $95 \% \pm 3 \%$, respectively), whereas protection with constant-flow esmolol cardioplegic infusion was significantly decreased at ischemic durations longer than 60 minutes. This decrease in efficacy of constant-flow esmolol cardio- 
plegia was associated with increasing coronary perfusion pressure leading to myocardial injury.

Conclusions: Oxygenation of esmolol cardioplegia (Krebs-Henseleit buffer plus 1.0 $\mathrm{mmol} / \mathrm{L}$ esmolol) was essential for optimal myocardial protection. Multidose infusion of oxygenated esmolol cardioplegia provided good myocardial protection during extended periods of normothermic ischemia. Esmolol cardioplegia may provide an efficacious alternative to hyperkalemia.

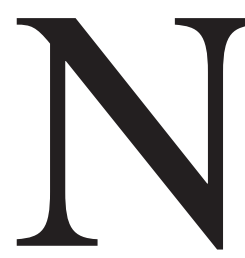

umerous experimental and clinical studies have demonstrated that $\beta$-adrenergic antagonists $(\beta$-blockers) can attenuate the extent of myocardial injury during ischemia and reperfusion, ${ }^{1-4}$ although the mechanism of the cardioprotective efficacy of these drugs during myocardial ischemia is not completely understood. Possible mechanisms include decreasing heart rate and contractility (with a reduction of myocardial oxygen consumption), ${ }^{5}$ decreasing sympathetic tone, ${ }^{6}$ altering myocardial substrate use, ${ }^{7}$ stabilizing cell membranes, ${ }^{8}$ and reducing lipid peroxidation of membrane phospholipids. ${ }^{9}$ The utility of $\beta$-blockers, however, is limited by their negative inotropic and chronotropic properties, and life-threatening adverse effects related to prolonged $\beta$-blockade (eg, shock, bradycardia, bronchospasm, pulmonary edema, and complete heart block) remain a clinical problem.

To minimize the risks of adverse effects related to prolonged $\beta$-blockade therapy in the critically ill patient, a novel group of ultra-short-acting, titratable $\beta$-blockers were designed. ${ }^{10,11}$ Esmolol, the first such intravenous $\beta$-blocker, was developed to produce rapid and titratable $\beta$-adrenergic blockade with minimal adverse effects. Esmolol is an ultrashort-acting, cardioselective $\beta$-blocker that is rapidly hydrolyzed by an esterase in blood cell cytosol ${ }^{12}$ to form methanol and an acid metabolite (ASL-8123) that is essentially inactive as a $\beta$-blocker. ${ }^{13}$ Esmolol has also been shown to protect the heart during cardiac surgery; Sweeney and Frazier $^{14}$ were the first to use esmolol in patients with compromised left ventricular function. High doses of esmolol were intravenously infused during continuous perfusion of the coronary arteries with normothermic blood to suppress cardiac inotropy and chronotropy, such that the heart rate was slowed sufficiently (without inducing cardiac arrest) to perform the operation. Since then, experimental and clinical studies ${ }^{15-17}$ have shown that the induction by high-dose esmolol of adequate cardiac surgical conditions has provided myocardial preservation equivalent to or better than cold crystalloid or blood cardioplegia. With this technique, however, which consists of continuous coronary infusion of blood containing esmolol, the surgeon is not provided with a still and blood-free operating field. There have been a few case reports from surgeons ${ }^{18,19}$ noting that high-dose esmolol can arrest the heart when the aorta is calcified and that aortic crossclamping should be avoided.

Previously, we ${ }^{20}$ showed that multidose infusion of esmolol cardioplegia in oxygenated buffer completely protected the myocardium during 40 minutes of normothermic $\left(37^{\circ} \mathrm{C}\right)$ global ischemia in an isolated rat heart preparation. Moreover, $w^{20}$ demonstrated that continuous infusion of esmolol cardioplegia (inducing cardiac arrest) failed to completely protect myocardial function, despite avoidance of ischemia, and was significantly less protective than multidose infusion of esmolol cardioplegia during global ischemia. This study was therefore conducted to investigate the importance of oxygenation in the protective effect of multidose esmolol cardioplegia; in addition, we examined the efficacy of multidose esmolol cardioplegic infusions during prolonged ischemia and sought to determine whether the method of infusion influenced this protection.

\section{Material and Methods}

\section{Animals}

Adult male Wistar rats (240-300 g body weight; Bantin and Kingman, Hull, UK) were used. All animals received humane care in accordance with the "Guidance on the Operation of the Animals (Scientific Procedures) Act of 1986" published by Her Majesty's Stationary Office, London, United Kingdom. Rats were anesthetized with $95 \%$ oxygen $/ 5 \%$ carbon dioxide bubbled through diethyl ether; they were then anticoagulated with intravenously administered heparin (1000 IU/kg body weight).

\section{Heart Isolation and Perfusion}

Hearts were rapidly excised from the anesthetized rats and immersed in cold $\left(4^{\circ} \mathrm{C}\right)$ Krebs-Henseleit bicarbonate buffer (KHB). The aorta was then cannulated, and the heart was perfused in the Langendorff mode as previously described elsewhere. ${ }^{21}$ A unipolar electrocardiogram was obtained through a silver electrode inserted into the free wall of the left ventricle and a reference electrode connected to the aortic cannula; the electrocardiogram was continuously recorded throughout the protocol. All hearts were subjected to an equilibration period of aerobic perfusion for $20 \mathrm{~min}$ utes, and baseline readings of left ventricular systolic pressure (in 
Study $1 \mathrm{~A}$.

(i) Oxy-KHB

\begin{tabular}{|l|l|l|l|l|l|l|}
\hline 20 & 3 & 3 & 3 & 3 & 60 \\
\hline Perfusion & 12 & 12 & 12 & 15 & Reperfusion \\
\hline
\end{tabular}

(ii) Deoxy-KHB

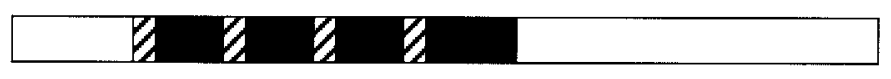

(iii) Oxy-Esmolol

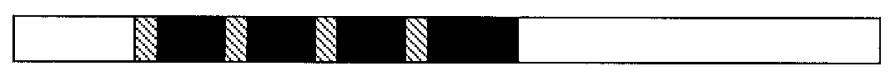

(iv) Deoxy-Esmolol

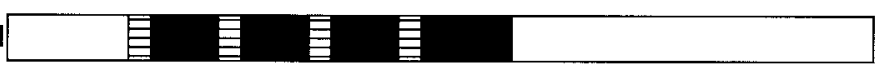

Study $1 B$.

(v) STH2

\begin{tabular}{|c|c|c|c|c|c|}
\hline 20 & & 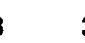 & 3 & & 60 \\
\hline \begin{tabular}{|l||} 
Perfusion \\
\end{tabular} & 12 & 12 & 12 & 15 & Reperfusion \\
\hline
\end{tabular}

(vi) Oxy-STH2

(vii) Oxy-Esmolol

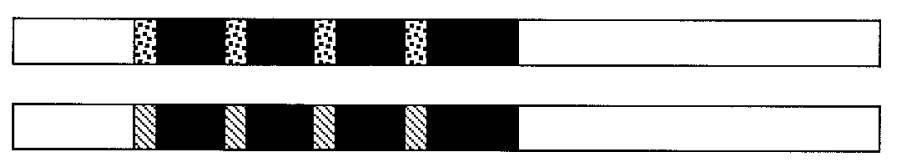

Study 2.

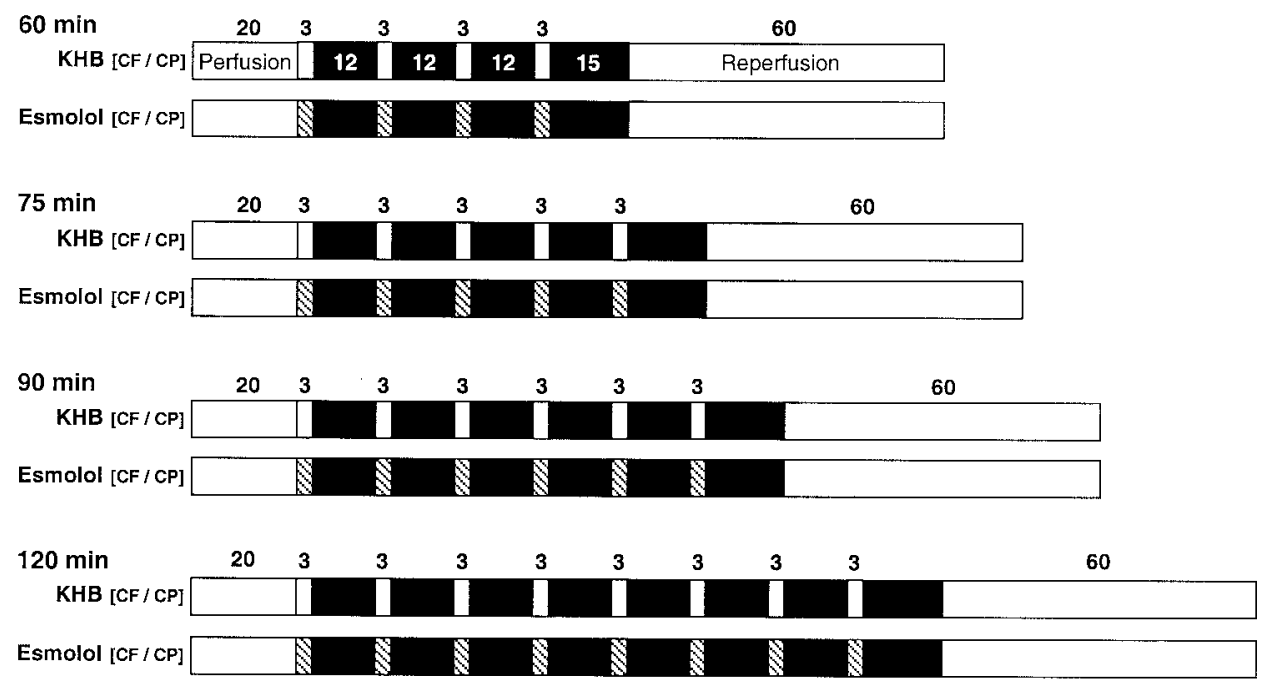

Figure 1. For legend see opposite page.

millimeters of mercury), left ventricular end-diastolic pressure (LVEDP; in millimeters of mercury), heart rate (in beats per minute), and coronary flow (in milliliters per minute) were then taken. Left ventricular developed pressure (LVDP) was calculated as left ventricular systolic pressure minus LVEDP. Perfusion pressure was monitored continuously through a sidearm of the aortic cannula by means of a second pressure transducer. Coronary flow was measured by timed collection of the coronary effluent. Infusion volume of cardioplegic solution was measured by collecting coronary effluent during infusion of cardioplegic solution.

\section{Exclusion Criteria}

Hearts not satisfying previously assigned inclusion criteria at the time of the baseline readings (after 20 minutes of aerobic perfusion) were excluded from the study. The acceptable ranges for LVDP, heart rate, and coronary flow were greater than $100 \mathrm{~mm}$ $\mathrm{Hg}$, greater than 220 beats/min, and 8 to $16 \mathrm{~mL} / \mathrm{min}$, respectively.

\section{Perfusion Medium}

The perfusion medium was a modified KHB with the following composition: 118.5-mmol/L sodium chloride, 25.0-mmol/L so- 
dium hydrogen carbonate, $4.8-\mathrm{mmol} / \mathrm{L}$ potassium chloride, $1.2-$ $\mathrm{mmol} / \mathrm{L}$ magnesium sulfate, $1.2-\mathrm{mmol} / \mathrm{L}$ potassium dihydrogen orthophosphate, $1.4-\mathrm{mmol} / \mathrm{L}$ calcium chloride, and 11.0-mmol/L glucose. The buffer was prepared daily and filtered through a 5- $\mu \mathrm{m}$ pore size cellulose nitrate membrane filter before use. Oxygenated KHB (oxy-KHB) was continuously gassed with a $95 \%$ oxygen $/ 5 \%$ carbon dioxide mixture to yield a $\mathrm{pH}$ of 7.4 at $37^{\circ} \mathrm{C}$. For deoxygenated KHB (deoxy-KHB), the buffer was continuously gassed with a $95 \%$ nitrogen $/ 5 \%$ carbon dioxide mixture so that a $\mathrm{pH}$ of 7.4 at $37^{\circ} \mathrm{C}$ was maintained.

\section{Preparation and Administration of Esmolol and St} Thomas' Hospital Cardioplegic Solutions

Esmolol (Brevibloc; Baxter Pharmaceuticals, Crowthorne, Berkshire, UK) was provided in vials containing $10 \mathrm{~mL}$ of a 250 $\mathrm{mg} / \mathrm{mL}$ solution. A $1.0-\mathrm{mmol} / \mathrm{L}$ esmolol solution (which we have previously shown to be the optimal concentration to achieve cardiac arrest ${ }^{20}$ ) was prepared by adding $1.5 \mathrm{~mL}$ of the concentrated esmolol solution to $1000 \mathrm{~mL}$ of oxy-KHB. St Thomas' Hospital cardioplegic solution No. 2 (STH2) had the following composition: $110.0-\mathrm{mmol} / \mathrm{L}$ sodium chloride, $16.0-\mathrm{mmol} / \mathrm{L}$ magnesium chloride hexahydrate, $16.0-\mathrm{mmol} / \mathrm{L}$ potassium chloride, 1.2$\mathrm{mmol} / \mathrm{L}$ calcium chloride dihydrate, and $10.0-\mathrm{mmol} / \mathrm{L}$ sodium hydrogen carbonate. STH2 was prepared daily, adjusted to $\mathrm{pH} 7.8$ when at $37^{\circ} \mathrm{C}$, and then filtered through a $5-\mu \mathrm{m}$ cellulose nitrate membrane filter before use. These solutions were delivered at $37^{\circ} \mathrm{C}$, either at constant pressure of $45 \mathrm{~mm} \mathrm{Hg}$ or at a constant flow rate of $14 \mathrm{~mL} / \mathrm{min}$ for 3 minutes each infusion. Normothermic $\left(37^{\circ} \mathrm{C}\right)$ crystalloid cardioplegia was used in preference to hypo- thermic crystalloid cardioplegia to avoid the additional protection associated with hypothermia and thus measure the protective effect of the cardioplegia itself.

\section{Perfusion Protocol}

In all perfusion protocols, each heart was supplied with KHB from a temperature-regulated reservoir $\left(37^{\circ} \mathrm{C}\right)$ at a constant perfusion pressure equivalent to $75 \mathrm{~mm} \mathrm{Hg}$ before and after global ischemia. After equilibration, hearts were subjected to one of three perfusion protocols, as shown in Figure 1. During the ischemic period, hearts were immersed in $\mathrm{KHB}$ at $37^{\circ} \mathrm{C}$ (using a temperature-controlled, water-jacketed heart chamber).

Study 1A: Effects on myocardial protection of oxygenation during multidose infusion of esmolol cardioplegia. Hearts were randomly assigned to one of four groups ( $\mathrm{n}=6$ per group): (i) oxy-KHB, multidose oxy-KHB infused for 3 minutes at $14 \mathrm{~mL} /$ min before and every 15 minutes during 60 minutes of intermittent infusion and global ischemia; (ii) deoxy-KHB, multidose deoxyKHB infused for 3 minutes at $14 \mathrm{~mL} / \mathrm{min}$ before and every 15 minutes during 60 minutes of intermittent infusion and global ischemia; (iii) oxyesmolol, multidose oxygenated (95\% oxygen $/ 5 \%$ carbon dioxide) esmolol infused for 3 minutes at 14 $\mathrm{mL} / \mathrm{min}$ before and every 15 minutes during 60 minutes of intermittent infusion and global ischemia; and (iv) deoxyesmolol, multidose deoxygenated ( $95 \%$ nitrogen $/ 5 \%$ carbon dioxide) esmolol infused for 3 minutes at $14 \mathrm{~mL} / \mathrm{min}$ before and every 15 minutes during 60 minutes of intermittent infusion and global ischemia. All protocols were followed by a further 60 minutes of reperfusion,

\begin{abstract}
Figure 1. Experimental perfusion protocols. In all protocols, hearts were perfused with KHB at constant pressure equivalent to $75 \mathrm{~mm} \mathrm{Hg}$ both before and after ischemia. Study 1A, Hearts were randomly assigned to one of four groups ( $n=6$ per group): oxy-KHB (i), multidose oxygenated ( $95 \%$ oxygen/5\% carbon dioxide) KHB infused for 3 minutes at $14 \mathrm{~mL} / \mathrm{min}$ before and every 15 minutes during 60 minutes of intermittent infusion and global ischemia; deoxy-KHB (ii), multidose deoxygenated (95\% nitrogen/5\% carbon dioxide) KHB infused for 3 minutes at $14 \mathrm{~mL} / \mathrm{min}$ before and every 15 minutes during 60 minutes of intermittent infusion and global ischemia; oxyesmolol (iii), multidose oxygenated ( $95 \%$ oxygen $/ 5 \%$ carbon dioxide) esmolol infused for 3 minutes at $14 \mathrm{~mL} / \mathrm{min}$ before and every 15 minutes during 60 minutes of intermittent infusion and global ischemia; and deoxyesmolol (iv), multidose deoxygenated ( $95 \%$ nitrogen/5\% carbon dioxide) esmolol infused for 3 minutes at $14 \mathrm{~mL} / \mathrm{min}$ before and every 15 minutes during $\mathbf{6 0}$ minutes of intermittent infusion and global ischemia. All protocols were followed by $\mathbf{6 0}$ further minutes of reperfusion, when recovery of myocardial function was measured. Study 1B, Hearts were randomly assigned to one of three groups ( $n=6$ per group): STH2 (v), multidose normoxic (equilibrated with room air) STH2 infused for 3 minutes at $\mathbf{4 5 ~} \mathrm{mm}$ Hg before and every $\mathbf{1 5}$ minutes during $\mathbf{6 0}$ minutes of intermittent infusion and global ischemia; oxy-STH2 (vi), multidose oxygenated (95\% oxygen/5\% carbon dioxide) STH2 infused for 3 minutes at 45 $\mathbf{m m ~ H g}$ before and every 15 minutes during $\mathbf{6 0}$ minutes of intermittent infusion and global ischemia; and oxyesmolol (vii), multidose oxygenated (95\% oxygen/5\% carbon dioxide) esmolol infused for 3 minutes at $45 \mathrm{~mm} \mathbf{~} \mathrm{gg}$ before and every 15 minutes during 60 minutes of intermittent infusion and global ischemia. All protocols were followed by 60 further minutes of reperfusion, when recovery of myocardial function was measured. Study 2, Hearts were randomly assigned to one of four ischemic duration groups: $60,75,90$, and 120 minutes of ischemia. In addition, for each ischemic duration group, hearts were randomly assigned to four treatment groups ( $\mathbf{n}=6$ per group): multidose KHB infused at constant flow (CF) for 3 minutes at $14 \mathrm{~mL} / \mathrm{min}$ before and every 15 minutes during intermittent infusion and global ischemia, multidose KHB infused at constant pressure (CP) for 3 minutes at 45 mm $\mathrm{Hg}$ before and every 15 minutes during intermittent infusion and global ischemia, multidose esmolol cardioplegia infused at constant flow for 3 minutes at $14 \mathrm{~mL} / \mathrm{min}$ before and every 15 minutes during intermittent infusion and global ischemia, and multidose esmolol cardioplegia infused at constant pressure for 3 minutes at $45 \mathrm{~mm} \mathrm{Hg} \mathrm{before}$ and every 15 minutes during intermittent infusion and global ischemia. All protocols were followed by $\mathbf{6 0}$ further minutes of reperfusion, when recovery of myocardial function was measured.
\end{abstract}


when recovery of myocardial function was measured (Figure 1, study 1A).

Study 1B: Comparison of myocardial protection by multidose St Thomas' Hospital cardioplegic solution No. 2 and esmolol cardioplegia. Hearts were randomly assigned to one of three groups ( $\mathrm{n}=6$ per group): (v) STH2, multidose normoxic (equilibrated with room air) STH2 infused for 3 minutes at $45 \mathrm{~mm} \mathrm{Hg}$ before and every 15 minutes during 60 minutes of intermittent infusion and global ischemia; (vi) oxy-STH2, multidose oxygenated (95\% oxygen $/ 5 \%$ carbon dioxide) STH 2 infused for 3 minutes at $45 \mathrm{~mm} \mathrm{Hg}$ before and every 15 minutes during 60 minutes of intermittent infusion and global ischemia; and (vii) oxyesmolol, multidose oxygenated (95\% oxygen/5\% carbon dioxide) esmolol infused for 3 minutes at $45 \mathrm{~mm} \mathrm{Hg}$ before and every 15 minutes during 60 minutes of intermittent infusion and global ischemia. All protocols were followed by a further 60 minutes of reperfusion, when recovery of myocardial function was measured (Figure 1, study 1B).

Study 2: Cardioprotective efficacy of multidose esmolol cardioplegia during prolonged ischemic durations. Hearts were randomly assigned to one of four ischemic duration groups: 60, 75, 90, and 120 minutes of ischemia. In addition, within each ischemic duration group, hearts were randomly assigned to four treatment groups ( $\mathrm{n}=6$ per group): constant-flow KHB, multidose KHB infused for 3 minutes at $14 \mathrm{~mL} / \mathrm{min}$ before and every 15 minutes during intermittent infusion and global ischemia; constant-pressure $\mathrm{KHB}$, multidose KHB infused for 3 minutes at $45 \mathrm{~mm} \mathrm{Hg}$ before and every 15 minutes during intermittent infusion and global ischemia; constant-flow esmolol, multidose esmolol cardioplegia infused for 3 minutes at $14 \mathrm{~mL} / \mathrm{min}$ before and every 15 minutes during intermittent infusion and global ischemia; and constantpressure esmolol, multidose esmolol cardioplegia infused for 3 minutes at $45 \mathrm{~mm} \mathrm{Hg}$ before and every 15 minutes during intermittent infusion and global ischemia. All protocols were followed by a further 60 minutes of reperfusion, when recovery of myocardial function was measured (Figure 1, study 2).

\section{Expression of Results}

Postischemic recoveries of LVDP, heart rate, and coronary flow were expressed as percentage of baseline values at the end of 20 minutes of aerobic perfusion; LVEDP was expressed as an absolute value (in millimeters of mercury). In addition, changes in infusion pressure or infusion volume during drug infusion were expressed as absolute values (in millimeters of mercury or milliliters per minute).

\section{Statistical Analysis}

Statistical analysis was performed with StatView and SuperANOVA (SAS Institute, Inc, Cary, NC) on an Apple Macintosh computer (Apple Computer, Inc, Cupertino, Calif). All data are reported as mean \pm SEM. Comparisons between groups were assessed for significance by 1-way analysis of variance with post hoc analysis with the Fisher protected least significant difference test, which allowed multiple comparisons (or the Scheffé test when appropriate). The Student paired $t$ test was used to compare paired means.

\section{Results}

Study 1A: Effects on Myocardial Protection of Oxygenation During Multidose Infusion of Esmolol Cardioplegia

The mean baseline values for LVDP, coronary flow, heart rate, and LVEDP after 20 minutes of aerobic perfusion are shown in Table 1. There were no significant differences between groups in any of these values.

\section{Recovery of function}

LEFT VENTRICULAR DEVELOPED PRESSURE, CORONARY FLOW, AND HEART RATE. The changes in recovery of LVDP during 60 minutes of reperfusion after 60 minutes of intermittent global ischemia are shown in Figure 2, A. Hearts subjected to multidose infusions of deoxy-KHB had almost no recovery, whereas those subjected to oxy-KHB recovered rapidly to a plateau value around $60 \%$ by 20 minutes. Addition of $1 \mathrm{mmol} / \mathrm{L}$ esmolol to either oxy-KHB or deoxy-KHB provided significant additional protection, improving recovery by approximately $40 \%$ in either case (Figure 2, A, Table 1), although the rate at which this was achieved was much slower (reaching a plateau by 30 minutes) in the deoxyesmolol group (Figure 2, A). Interestingly, oxygenation improved recovery by approximately $50 \%$ in each case, indicating the highly significant impact of oxygenation of these solutions.

Recovery of coronary flow (after 60 minutes of intermittent global ischemia) throughout 60 minutes of reperfusion is shown in Figure 3, A. Similar values were observed in both deoxygenated groups, reaching $60 \%$ by 5 minutes and only increasing slowly to $70 \%$ to $80 \%$ by 60 minutes. In contrast, both oxygenated groups exhibited a hyperemic response (around $110 \%$ recovery) at 5 minutes, and this was sustained in the oxyesmolol group but decreased in the oxy-KHB group to similar levels as the deoxygenated groups (Figure 3, A). Recovery of heart rate (Table 1) was similar in all groups and hence did not appear to be related to oxygenation.

LEFT VENTRICULAR END-DIASTOLIC PRESSURE. Essentially, LVEDP recovery mirrored the results seen for LVDP; thus, high values of LVEDP were maintained in the deoxygenated groups, with lower values in the oxygenated groups. The addition of esmolol to each solution was associated with a beneficial decrease in LVEDP.

INFUSION PRESSURE OF CONSTANT-FLOW INFUSIONS DURING ISCHEMIA. Infusion pressures at each of the four infusions (before and every 15 minutes throughout ischemia) remained relatively constant for the oxygenated solutions but increased significantly with each infusion of the deoxygenated solutions (Figure 4, A).

\section{Study 1B: Comparison of Myocardial Protection by Multidose STH2 and Esmolol Cardioplegia}

The mean baseline values for LVDP, coronary flow, heart rate, and LVEDP after 20 minutes of aerobic perfusion are 
TABLE 1. Baseline values (measured after equilibration of 20 minutes aerobic perfusion) and percentage recovery (after 60 minutes of intermittent global ischemia and 60 minutes of reperfusion) of LVDP, coronary flow, heart rate, and LVEDP

\begin{tabular}{lcccc}
\hline & Oxy-KHB & Deoxy-KHB & 0xyesmolol & Deoxyesmolol \\
\hline LVDP (mm Hg) & $125 \pm 14$ & $119 \pm 12$ & $129 \pm 8$ & $111 \pm 6$ \\
Recovery (\%) & $58.3 \pm 9.0^{*}$ & $4.4 \pm 1.5$ & $97.2 \pm 4.7 \dagger$ & $44.7 \pm 7.6^{*}$ \\
Coronary flow (mL/min) & $13.2 \pm 0.6$ & $11.2 \pm 0.8$ & $12.2 \pm 0.7$ & $11.4 \pm 0.3$ \\
Recovery (\%) & $61.3 \pm 2.3$ & $77.8 \pm 10.8$ & $107.5 \pm 13.9 \pm$ & $66.6 \pm 5.1$ \\
Heart rate (beats/min) & $273 \pm 7$ & $269 \pm 12$ & $245 \pm 7$ & $265 \pm 8$ \\
Recovery (\%) & $95.6 \pm 2.9$ & $104.9 \pm 5.6$ & $107.4 \pm 7.2$ & $8.5 \pm 3.9$ \\
LVEDP (mm Hg) & $5.8 \pm 1.6$ & $7.0 \pm 1.0$ & $5.7 \pm 1.3$ & $6.7 \pm 0.6$ \\
Final LVEDP (mm Hg) & $29.8 \pm 8.4$ & $72.3 \pm 4.1 \dagger$ & $8.8 \pm 3.3 \dagger$ & $42.7 \pm 4.4$ \\
\hline
\end{tabular}

The differences between groups in baseline values could be due to chance $(P>.2)$.

${ }^{*} P<.05$ versus deoxy-KHB.

$\dagger P<.01$ versus other groups.

$\ddagger P<.05$ versus other groups.

shown in Table 2. There were no significant differences between groups in any of these values.

\section{Recovery of function}

LEFT VENTRICULAR DEVELOPED PRESSURE, CORONARY FLOW, AND HEART RATE. The recovery profiles of LVDP after 60 minutes of intermittent global ischemia (expressed as a percentage of preischemic baseline value) are shown for each group in Figure 2, $B$. Hearts from all groups recovered rapidly and reached a plateau level within 5 to 20 minutes of reperfusion. As before, hearts infused with multidose esmolol cardioplegia recovered extremely rapidly to preischemic values, which were significantly higher than the STH2 groups. Interestingly, there were no differences in recovery between hearts subjected to oxy-STH2 and normoxic STH2. Recovery of coronary flow during 60 minutes of reperfusion after 60 minutes of intermittent global ischemia (Figure 3, $B$ ) was similar to that in the previously described study (study 1A). Thus recovery of coronary flow in hearts subjected to multidose oxygenated esmolol was maintained at values that were significantly higher (approximately 120\%) than preischemic baseline values $(P<.05$ vs preischemic value). In contrast, hearts from both STH2 groups had similar patterns of recovery, with coronary flow reaching a hyperemic peak at 5 minutes of reperfusion followed by a subsequent decline throughout the remaining reperfusion period.

Recovery of heart rate (Table 2) was similar in all groups, with no significant differences among all groups.

LEFT VENTRICULAR END-DIASTOLIC PRESSURE. Recovery of LVEDP in all groups was similar to the corresponding preischemic value. There were no differences between groups throughout the reperfusion period.

INFUSION VOLUME OF CONSTANT PRESSURE-INFUSIONS DURING ISCHEMIA. Infusion volume at each infusion (before and every 15 minutes throughout ischemia) remained relatively constant for both STH2 groups (Figure 4, B), although oxy-STH2 group volumes were significantly higher than those of STH2 and oxyesmolol groups. Interestingly, the volume in the oxyesmolol group decreased significantly at the second infusion but remained constant thereafter.

\section{Study 2: Cardioprotective Efficacy of Multidose Esmolol Cardioplegia During Prolonged Ischemic Durations}

We have shown that multiple infusions of oxygenated esmolol cardioplegia completely protected hearts subjected to 45 minutes $^{20}$ and 60 minutes (this study) of $37^{\circ} \mathrm{C}$ global ischemia, regardless of the method of infusion (constant flow or constant perfusion pressure). This study was therefore conducted to investigate the protective efficacy of multidose esmolol cardioplegic infusions during prolonged ischemia and also to determine whether the method of infusion influences this protection.

The mean baseline values of LVDP ranged from $105.2 \pm$ 4.8 to $129.0 \pm 8.4 \mathrm{~mm} \mathrm{Hg}$ in all groups; there were no significant differences between groups. Similarly, coronary flow ranged from $9.3 \pm 0.3$ to $12.0 \pm 1.5 \mathrm{~mL} / \mathrm{min}$ and heart rate ranged from $253.7 \pm 12.0$ to $283.5 \pm 7.2$ beats $/ \mathrm{min}$, with no significant differences between groups.

\section{Recovery of function}

LEFT VENTRICULAR DEVELOPED PRESSURE, CORONARY FLOW, AND HEART RATE. Postischemic recovery of LVDP with increasing duration of global ischemia is shown in Figure 5, $A$. Hearts subjected to multidose esmolol cardioplegia infused at constant pressure had complete recovery (approximately $100 \%$ of preischemic values) for as long as 90 minutes of $37^{\circ} \mathrm{C}$ global ischemia and only decreased to $60.5 \% \pm 4.9 \%$ after 120 minutes of $37^{\circ} \mathrm{C}$ global ischemia. In hearts in which esmolol cardioplegia was infused at constant flow, however, although recovery was $98.4 \% \pm$ $5.2 \%$ after 60 minutes of $37^{\circ} \mathrm{C}$ global ischemia (confirming the results obtained in study $1 \mathrm{~A}$ ), recovery had decreased dramatically to only $40.7 \% \pm 4.3 \%$ by 75 minutes of $37^{\circ} \mathrm{C}$ global ischemia. Surprisingly, further increases in ischemic 

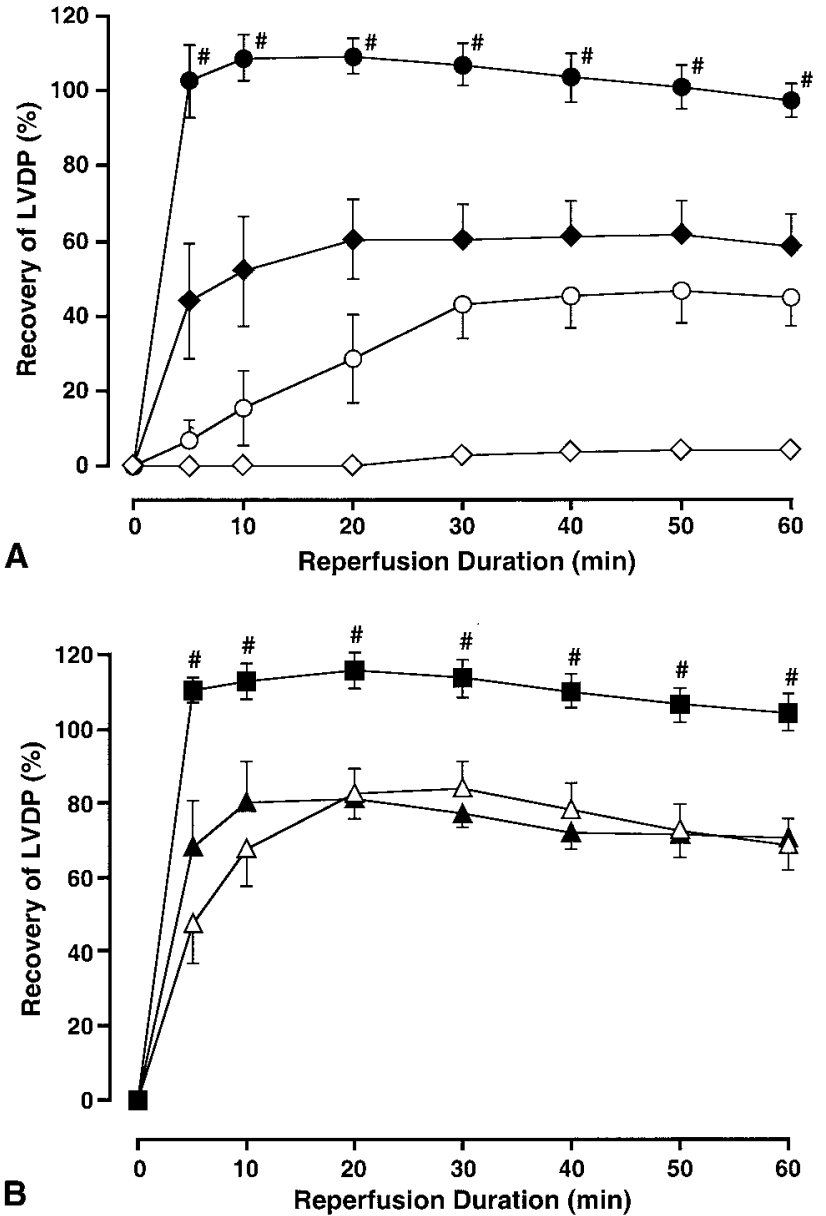

Figure 2. Recovery of LVDP (expressed as percentage of preischemic control value) throughout 60 minutes of reperfusion. A, Study 1A. Filled diamonds represent oxy-KHB; open diamonds represent deoxy-KHB; filled circles represent oxygenated esmolol (infused at $14 \mathrm{~mL} / \mathrm{min}$ ); open circles represent deoxygenated esmolol. B, Study 1B. Open triangles represent STH2; filled triangles represent oxy-STH2; filled squares represent oxygenated esmolol (infused at $45 \mathrm{~mm} \mathrm{Hg}$ ). Data points represent mean; error bars represent SEM; $\mathbf{n}=\mathbf{6}$ hearts/group. Pound sign indicates $P<.01$ versus all other groups.

duration (90 and 120 minutes) did not result in further decreases (with recoveries of $39.2 \% \pm 5.6 \%$ and $29.1 \% \pm$ $6.6 \%$, respectively).

Recovery of hearts subjected to multidose KHB infusion, either constant flow or constant pressure, decreased in a linear fashion with increasing duration of ischemia. Myocardial protection was significantly less than that with constant-pressure esmolol cardioplegia but was similar to that of constant-flow esmolol cardioplegia at all ischemic durations except 60 minutes.

Recovery of coronary flow in the esmolol cardioplegiatreated hearts was similar to that of LVDP. Hearts treated
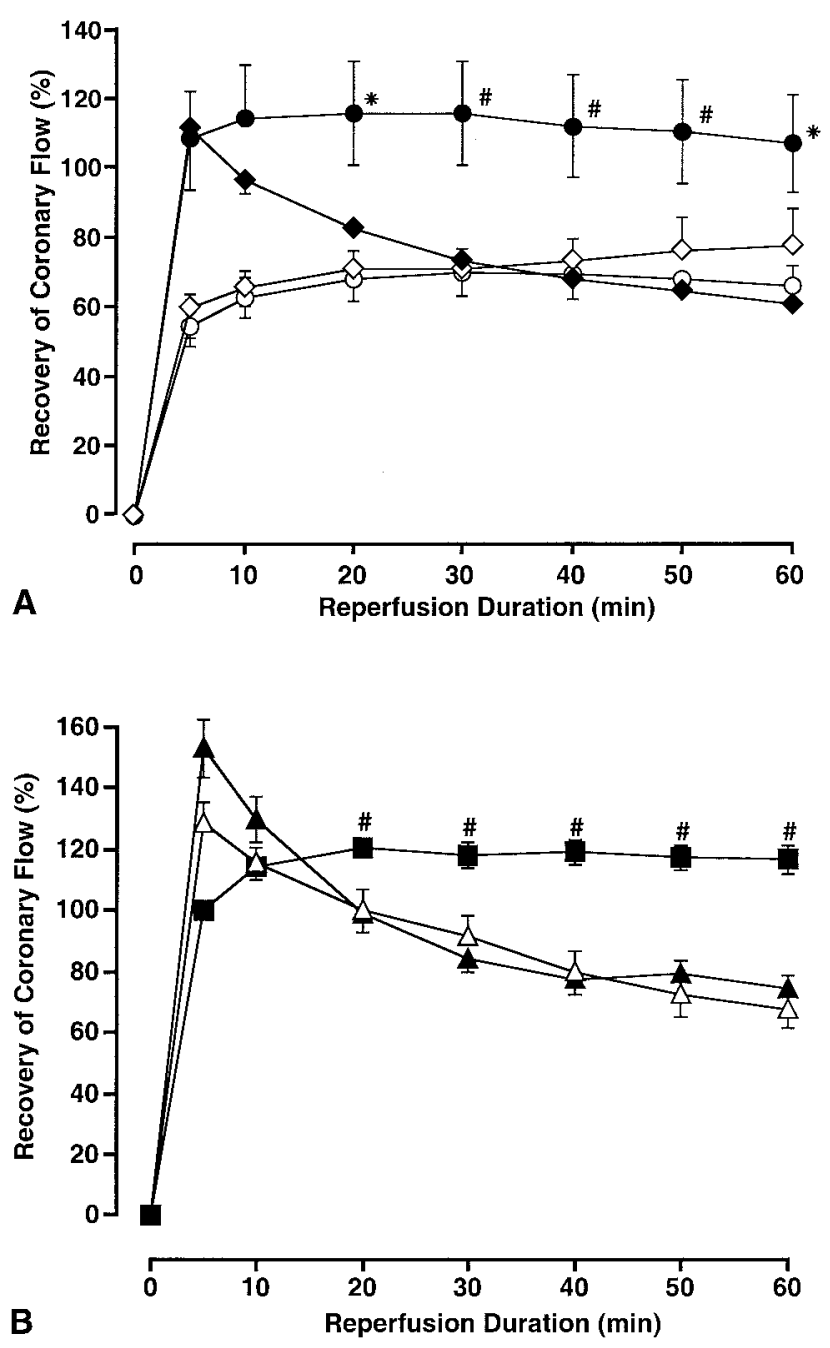

Figure 3. Recovery of coronary flow (expressed as percentage of preischemic control value) throughout $\mathbf{6 0}$ minutes of reperfusion. A, Study 1A. Filled diamonds represent oxy-KHB; open diamonds represent deoxy-KHB; filled circles represent oxygenated esmoIol (infused at $14 \mathrm{~mL} / \mathrm{min}$ ); open circles represent deoxygenated esmolol. B, Study 1B. Open triangles represent STH2; filled triangles represent oxy-STH2; filled squares represent oxygenated esmolol (infused at $45 \mathrm{~mm} \mathrm{Hg}$ ). Data points represent mean; error bars represent SEM; $\mathbf{n}=\mathbf{6}$ hearts/group. Asterisk indicates $\boldsymbol{P}<$ .05 versus all other groups; pound sign indicates $\boldsymbol{P}<.01$ versus all other groups.

with KHB had a relatively constant recovery of coronary flow of around $80 \%$ after all ischemic durations.

Surprisingly, the postischemic recovery of heart rate was similar in all groups but tended to be slightly higher in the KHB-treated hearts than in the esmolol cardioplegia-treated hearts.

LEFT VENTRICULAR END-DIASTOLIC PRESSURE. Changes in postischemic LVEDP (after 60 minutes of reperfusion) with increasing ischemic durations are shown in Figure $5, B$. In 
TABLE 2. Baseline values (measured after equilibration of 20 minutes aerobic perfusion) and percentage recovery (after $\mathbf{6 0}$ minutes of intermittent global ischemia and $\mathbf{6 0}$ minutes of reperfusion) of LVDP, coronary flow, heart rate, and LVEDP

\begin{tabular}{lccc}
\hline & STH2 & 0xy-STH2 & 0xyesmolol \\
\hline LVDP (mm Hg) & $114 \pm 5$ & $113 \pm 5$ & $119 \pm 7$ \\
Recovery (\%) & $68.6 \pm 6.9$ & $70.2 \pm 4.4$ & $104.3 \pm 5.1^{*}$ \\
Coronary flow (mL/min) & $11.9 \pm 1.5$ & $11.2 \pm 0.7$ & $10.9 \pm 0.7$ \\
Recovery (\%) & $67.3 \pm 5.7$ & $74.2 \pm 4.4$ & $116.7 \pm 4.6^{*}$ \\
Heart rate (beats/min) & $269 \pm 10$ & $262 \pm 11$ & $260 \pm 5$ \\
Recovery (\%) & $104.9 \pm 3.9$ & $102.6 \pm 3.8$ & $94.8 \pm 4.8$ \\
LVEDP (mm Hg) & $6.9 \pm 1.3$ & $5.3 \pm 0.7$ & $4.7 \pm 0.8$ \\
Final LVEDP (mm Hg) & $7.8 \pm 3.7$ & $6.2 \pm 1.1$ & $11.0 \pm 1.8$
\end{tabular}

The differences between groups in baseline values could be due to chance $(P>$.2).

${ }^{*} P<.01$ versus other groups.

each group, postischemic LVEDP increased with increasing duration of ischemia. In hearts with constant-pressure esmolol cardioplegia, LVEDP was significantly lower than in other groups at 75 and 90 minutes of ischemia but had increased to similar values by 120 minutes of ischemia. In contrast, LVEDP increased dramatically between 60 and 75 minutes of ischemia in hearts with constant-flow esmolol cardioplegia but then remained relatively constant.

INFUSION VOLUME OF CONSTANT-PRESSURE INFUSION DURING ISCHEMIA. The infusion volumes at each infusion during ischemia of either KHB or esmolol during constant-pressure infusion are shown in Figure 6, A. Infusion volume decreased with each infusion in the hearts treated with esmolol cardioplegia, whereas infusion volume changes in the KHBtreated hearts were minimal.

INFUSION PRESSURE OF CONSTANT-FLOW INFUSIONS DURING ISCHEMIA. The infusion pressures at each infusion during ischemia of either KHB or esmolol during constant-flow infusions are shown in Figure 6, B. KHB-treated hearts had minimal changes in infusion pressure for each infusion; in contrast, infusion pressure in the hearts treated with esmolol cardioplegia increased at each infusion. This was particularly evident between the fourth (60 minutes) and fifth (75 minutes) infusions, associated with a significant change in postischemic recovery of LVDP (Figure 5, A).

\section{Discussion}

This study demonstrated that although oxygenation of an esmolol-based arresting solution (esmolol cardioplegia) was essential for complete myocardial protection, esmolol itself exerted a significant protective effect. In addition, multidose esmolol cardioplegia was also shown to provide myocardial protection superior to that of STH2 under the same conditions. Furthermore, recovery of hearts protected with multidose oxygenated esmolol cardioplegia infused at a con-
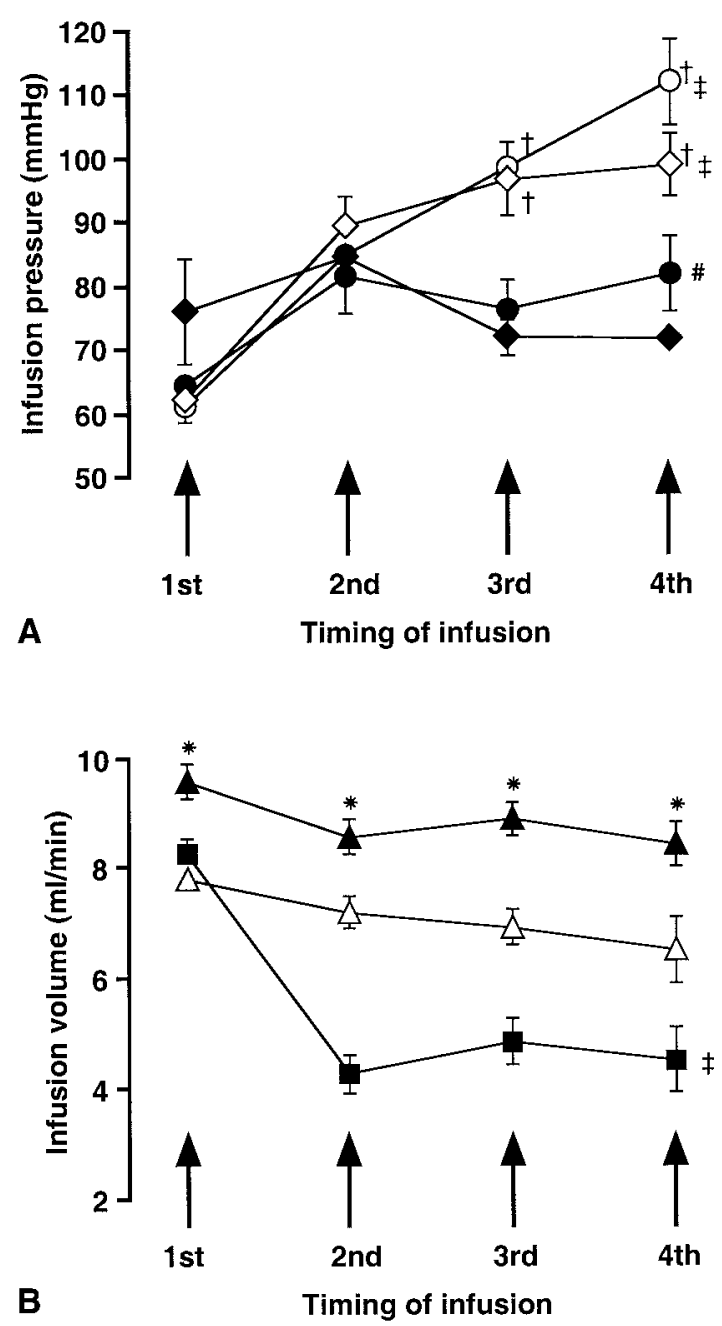

Figure 4. Changes during global ischemia in infusion pressure at each constant-flow infusion in study $1 A(A)$ and infusion volume at each constant-pressure infusion in study 1B (B). A, Study 1A. Filled diamonds represent oxy-KHB; open diamonds represent deoxy-KHB; filled circles represent oxygenated esmolol (infused at $14 \mathrm{~mL} / \mathrm{min}$ ); open circles represent deoxygenated esmolol. B, Study 1B. Open triangles represent STH2; filled triangles represent oxy-STH2; filled squares represent oxygenated esmolol (infused at $45 \mathrm{~mm} \mathrm{Hg}$ ). Data points represent mean; error bars represent SEM; $n=6$ hearts/group. Dagger indicates $P<.05$ versus oxy-KHB and oxyesmolol groups; double dagger indicates $\boldsymbol{P}<.01$ versus first infusion; pound sign indicates $\boldsymbol{P}<.05$ versus first infusion; asterisk indicates $\boldsymbol{P}<.05$ versus all other groups.

stant pressure during $37^{\circ} \mathrm{C}$ global ischemia remained at approximately $100 \%$ of preischemic values for as long as 90 minutes.
Multidose, Oxygenated Infusions and Myocardial Protection
Multidose infusion of hyperkalemic cardioplegia during global ischemia improves myocardial protection relative to 

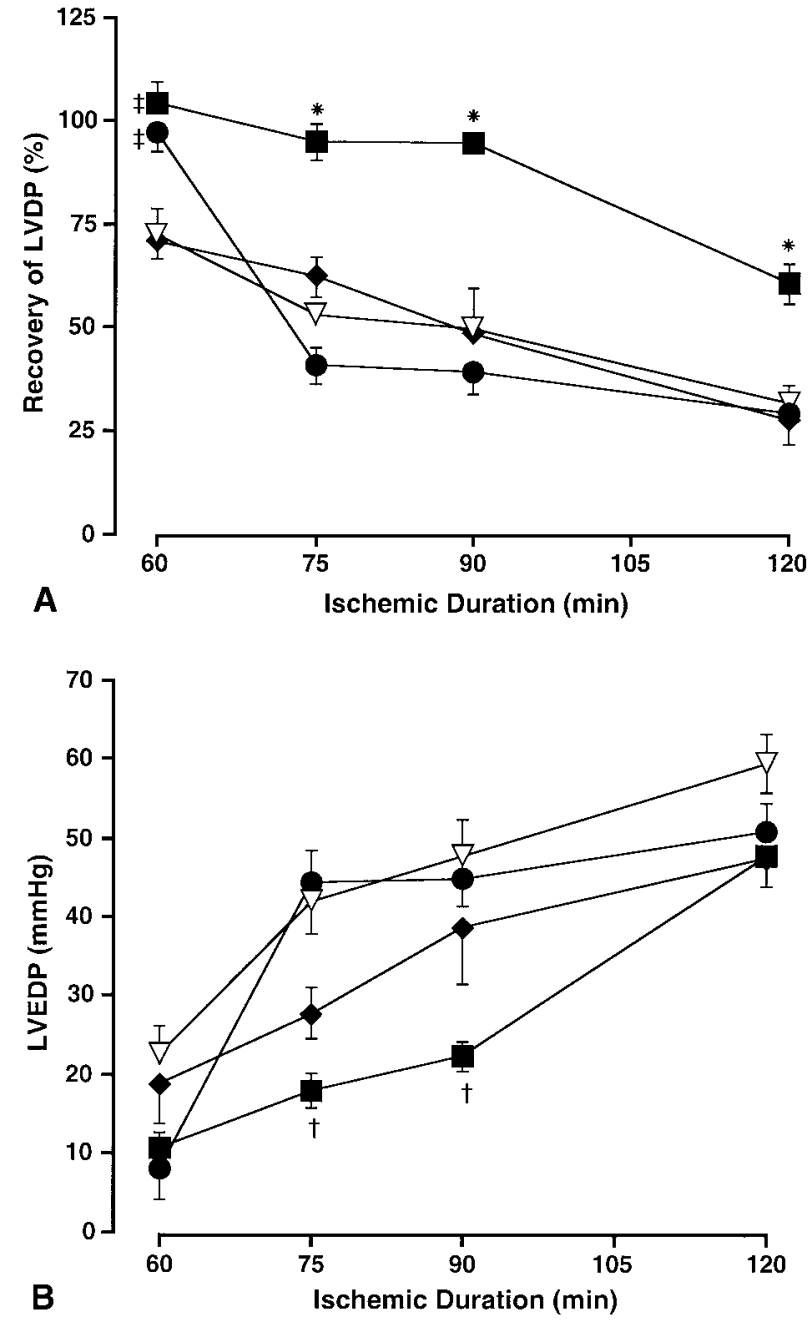

Figure 5. Recovery of LVDP ( $A$, expressed as percentage of preischemic control value) and LVEDP (B) with increasing durations of global ischemia (study 2). Filled diamonds represent KHB at constant flow; open inverted triangles represent KHB at constant pressure; filled circles represent esmolol at constant flow; and filled squares represent esmolol at constant pressure. Data points represent mean; error bars represent SEM; $n=6$ hearts/ group. Double dagger indicates $\boldsymbol{P}<.01$ versus KHB at constant flow and KHB at constant pressure; asterisk indicates $P<.01$ versus all other groups; dagger indicates $P<.05$ versus all other groups.

single-dose cardioplegia. ${ }^{22}$ Previously, we ${ }^{20}$ demonstrated that multidose infusions of oxygenated esmolol cardioplegia provided myocardial protection superior to that of a single infusion; therefore, in this study, all cardioplegic solutions were administered as multidose infusions. For normothermic ischemia, the initial cardioplegic infusion acts to arrest contraction (conserving energy); cellular metabolic activity will continue, however, and various anaerobic metabolites such as lactate and protons will accumulate in the tissue.
Subsequent cardioplegic infusions remove toxic metabolites, provide a small amount of oxygen for residual oxidative metabolism, and (with some solutions) provide substrates for anaerobic metabolism. In this study, the short half-life ( 9 minutes) of esmolol means that its pharmacologic effect will rapidly diminish, allowing the return of contractile activity; periodic replenishment of this solution would, therefore, be important to maintain diastolic arrest. A potential disadvantage of multidose cardioplegia under normothermic conditions might be the additional exposure of the myocardium to calcium, sodium, and water, thereby promoting tissue edema.

Clinically, intermittent ischemia (with or without fibrillation) and reperfusion continues to be used by some surgeons (particularly in the United Kingdom) with good results, ${ }^{23,24}$ and $\mathrm{we}^{21}$ previously demonstrated that intermittent global ischemia has an intrinsic myocardial protective effect equivalent to that of a crystalloid cardioplegia (STH2). That finding was confirmed by this study. Thus the beneficial effect observed with multidose esmolol is likely to be biphasic, involving both the protective effect of esmolol and that of intermittent reperfusion, and this supposition was confirmed by the results from the deoxy-KHB and deoxyesmolol groups in study 1A. Vascular washout (by intermittent reperfusion) removes harmful metabolic products (such as lactate, protons, or inorganic phosphate) from the myocardium, reducing the cumulative effects of these products during subsequent ischemia. In this study, however, hearts treated with multidose infusion of deoxyKHB (intermittent washout of metabolites only) had a poor recovery, suggesting that intermittent oxygenation rather than intermittent washout was of primary benefit. Furthermore, esmolol added to deoxy-KHB enhanced myocardial recovery to a value that was similar to that seen with oxy-KHB, confirming the independent protective effect of esmolol.

Most studies investigating the protective effect of oxygenation of crystalloid hyperkalemic cardioplegic solutions have demonstrated beneficial effects on the myocardium, both experimentally ${ }^{25,26}$ and clinically ${ }^{27}$; others, however, have been unable to demonstrate these beneficial effects. In this study (1B) we also failed to demonstrate an improvement in recovery of function with oxygenated $\mathrm{STH} 2$ relative to normoxic STH2; however, a number of differences between this and previous investigations may account for these effects. In this study $\mathrm{STH} 2$ was gassed with $95 \%$ oxygen $/ 5 \%$ carbon dioxide (which maintained the $\mathrm{pH}$ at $\mathrm{pH}$ 7.0), as was esmolol cardioplegia (which had a $\mathrm{pH}$ of 7.4 with this gas mixture). In contrast, previous studies $25,26,28$ used $100 \%$ oxygen in STH2, which caused a significant alkalosis and a depressed recovery of function. Although STH2 is usually adjusted to $\mathrm{pH} 7.8$ before use, experimental 
studies ${ }^{29,30}$ have demonstrated that an alkaline $\mathrm{pH}$ may not be optimal and that it should be used at neutral or slightly acidic $\mathrm{pH}$ values for optimal efficacy. In addition, when $100 \%$ oxygen was used, ${ }^{25,26,28} \mathrm{STH} 2$ and the ensuing ischemia were hypothermic $\left(10^{\circ} \mathrm{C}-20^{\circ} \mathrm{C}\right)$; this ought to favor a more alkaline $\mathrm{pH},{ }^{31}$ but it has been shown ${ }^{30}$ that $\mathrm{STH} 2$ only changes by 0.05 of a pH unit across the range of $10^{\circ} \mathrm{C}$ to $40^{\circ} \mathrm{C}$. Thus the $\mathrm{pH}$ values of the cardioplegic solutions used in this study were closer to the optimum value, and this may account for some of the differences between these studies and others. It is also possible that increased myocardial oxygen consumption at normothermia relative to delayed consumption at hypothermia (with the improved maintenance of high-energy phosphate compounds) may have influenced these differing results.

In contrast, it is obvious that oxygenation of esmolol cardioplegia plays an important role in its myocardial protection. Multidose infusion (every 15 minutes) of oxygenated esmolol (oxy-KHB containing $1 \mathrm{mmol} / \mathrm{L}$ esmolol) completely protected hearts during 60 minutes of $37^{\circ} \mathrm{C}$ global ischemia, whereas deoxygenated esmolol (gassed with $95 \%$ nitrogen $/ 5 \%$ carbon dioxide) was significantly less protective. However, deoxy-KHB gave almost no protection, indicating a significant protective effect for esmolol per se. It is possible that esmolol, by reducing myocardial oxygen demand, delays injury to the ischemic myocardium, and that reperfusion (reinfusion) with oxygenated esmolol cardioplegia at a time when the protected myocardium is still reversibly damaged may attenuate ischemic injury. If, on the other hand, reperfusion is initiated too late or anoxia continues, the myocardium is irreversibly injured, and esmolol fails to protect fully. Interestingly, Toleikis and Tomlinson $^{32}$ also demonstrated that acute $\beta$-blockade, both before and during a relatively short (30-minute) period of normothermic low-flow global ischemia in isolated rabbit hearts, was protective, whereas similar treatment through 60 minutes of ischemia was ineffective, supporting our suggestion that $\beta$-blockers are only likely to be protective during short periods of ischemia. Thus intermittent aerobic reperfusion may be required for esmolol cardioplegia to provide full myocardial protection.

\section{Method of Infusion: Constant Flow Versus Constant Pressure}

The direct vascular effects of esmolol were examined by Gorczynski ${ }^{13}$ in constant-flow-perfused hind limbs of pentobarbital-anesthetized, ganglion-blocked dogs. That study indicated that esmolol is devoid of direct vascular effects at doses that are within or above the $\beta$-blocking range but that extremely large doses may cause vasoconstriction. Murthy and Frishman ${ }^{33}$ showed that intravenous infusion of esmolol increased both mean coronary resistance and diastolic coronary resistance in dogs under barbiturate anesthesia. Our study confirmed these findings when esmolol cardioplegia
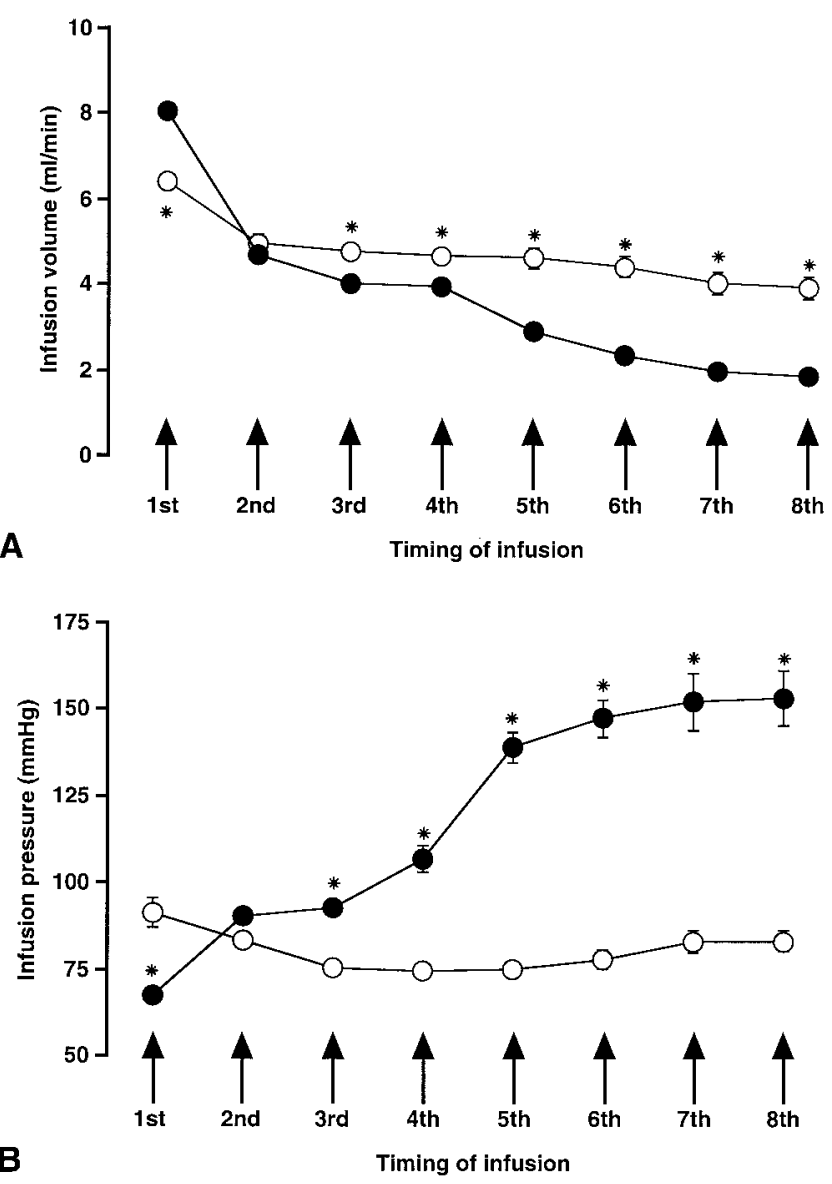

Figure 6. Changes during global ischemia in study 2 in infusion volume at each constant-pressure infusion $(A)$ and infusion pressure at each constant-flow infusion (B). Open circles represent KHB; filled circles represent esmolol. Data points represent mean; error bars represent SEM; $n=$ at least 6 hearts/group. Asterisk indicates $\boldsymbol{P}<.05$ versus esmolol in $A$, and $\boldsymbol{P}<.001$ versus KHB in B.

was infused at constant flow and perfusion pressure was monitored (Figure 6, B). Infusion pressure increased significantly at the fifth infusion and was closely correlated with a significant decrease in recovery of LVDP (Figure 5, $A$ ) and increased LVEDP (Figure 5, B); hence, this increased infusion pressure (coronary vascular resistance) appeared to be a cause of myocardial injury. Similarly, increasing numbers of infusions of esmolol cardioplegia at constant pressure resulted in a decline in infusion volume (reflecting the vasoconstrictive action of esmolol ${ }^{13}$ and associated with a considerably larger increase in coronary vascular resistance [data not shown] in both study 2 and study 1A), but that was not associated with a depressed recovery of function.

The lower coronary vascular resistance observed in constant-flow-infused hearts may be an artificial consequence of the infusion being forced through the vasculature (caus- 
ing vascular injury) and may lead to increased tissue edema, which would lead to deterioration in postischemic function. Although myocardial edema was not measured in this study, constant-pressure esmolol perfusion has previously been shown ${ }^{16}$ to minimize myocardial edema. We observed significantly lower LVEDP levels in hearts infused with esmolol cardioplegia at constant pressure (Figure 5, B), which would tend to support this suggestion.

\section{Possible Protective Mechanisms of Esmolol}

In this study, hearts infused ( 3 minutes every 15 minutes at constant pressure of $45 \mathrm{~mm} \mathrm{Hg}$ ) with oxygenated esmolol cardioplegia were completely protected for as long as 90 minutes of $37^{\circ} \mathrm{C}$ global ischemia. It is intriguing to speculate on how this could be achieved.

Initiation of ischemic arrest is associated with an accelerated release of endogenous catecholamines, producing a period of hypermetabolic function. ${ }^{34}$ This results in depletion of intracellular high-energy phosphate stores, which sharply reduces the tolerance of the cell to ischemia. ${ }^{32}$ The best available technique to maintain this tolerance involves reducing cell metabolism, thereby avoiding the period of hypermetabolic function and reducing the oxygen demands of the cell. We have previously shown ${ }^{20}$ that esmolol cardioplegia induces a rapid arrest (at a mean duration of around 50 seconds), which is similar to that of St Thomas' Hospital cardioplegia (around 35 seconds) and significantly shorter than ischemia alone (around 220 seconds). It is likely that part of the protective mechanism of esmolol cardioplegia involves maintenance of high-energy phosphates by rapid reduction of energy demands. $\beta$-Blockers, administered either before or just after ischemia, have been shown in numerous animal models ${ }^{1-4}$ to exert beneficial effects on ischemic myocardium. Although the mechanism for these benefits are unknown, speculations include improved balance between oxygen supply and demand by reducing oxygen demand through negative inotropic and chronotropic actions,, 535 increased oxygen supply as a result of increased blood flow to ischemic areas, ${ }^{35}$ and redistribution of blood flow from the subepicardium to the subendocardium. ${ }^{36}$ We also observed that hearts treated with oxygenated esmolol had a significantly higher coronary flow, which was sustained throughout the reperfusion period. This elevated coronary flow would considerably increase the available oxygen supply to the previously ischemic myocardium and thus represents a potential additional mechanism by which esmolol exerted its protective effect in this study. Thus multidose infusions of oxygenated esmolol cardioplegia may combine the protection that we previously observed with intermittent global ischemia and reperfusion $^{21}$ with that of rapid cardiac arrest (which decreases myocardial oxygen consumption), thereby enhancing overall protection.

\section{Limitations of This Study}

We concede that these studies were conducted in rat hearts perfused with a crystalloid solution rather than blood and that this is a relatively unphysiologic situation. We are aware that esmolol is metabolized by blood esterases ${ }^{12}$; consequently, this study would fail to reveal any interaction between the metabolism of esmolol and the cardioprotective efficacy of esmolol. It would be important to establish whether blood-based esmolol cardioplegia would be as effective as the crystalloid solutions used in these studies, and further studies are warranted.

It is also possible that esmolol may have adverse systemic effects that would not be revealed in our study; this would require investigation in the intact animal. Previous studies ${ }^{14,16,17}$ have used esmolol clinically during relatively long-term continuous infusions (albeit at lower concentrations), with no reports of any systemic adverse effects. Hence, we speculate that multiple, short, high-dose infusions are unlikely to have major systemic effects, although this would need verification.

Myocardial ischemic disease is a multifactorial process; there is a spectrum of injury that affects the method of myocardial protection. Hearts used in this study were taken from healthy rats, and it is likely that any protective effect of esmolol would be different in jeopardized hearts suffering from ischemic injury or disease. In addition, any such hearts are likely to require prolonged periods of ischemia to correct the lesion, and we have shown that multidose esmolol infusions are effective for relatively prolonged ischemic durations at $37^{\circ} \mathrm{C}$.

Although it is dangerous to extrapolate from experimental studies in the isolated rat heart to the clinical situation, there are a number of potential clinical benefits that might be available with the use of esmolol cardioplegia. The total volume of esmolol required to induce and maintain cardiac arrest is quite low (the addition of a suitable volume of esmolol to oxygenated blood to make up a $1 \mathrm{mmol} / \mathrm{L}$ concentration) relative to potassium cardioplegia, for which volumes in excess of $1000 \mathrm{~mL}$ are required. Consequently, additional hemodilution during cardiopulmonary bypass should be avoided with esmolol arrest, in contrast to warm continuous-infusion of blood cardioplegia or the cardiac surgical condition induced with esmolol added to blood for continuous infusion, ${ }^{14-16}$ multidose intermittent esmolol infusion would provide a still and blood-free operating field during warm (normothermic) heart surgery. This technique of multidose esmolol infusion can be used for coronary artery bypass surgery, or any other type of cardiac surgery.

\section{Conclusion}

Oxygenation of the esmolol cardioplegia was essential for optimal myocardial protection, and it was shown to be more efficacious than the well-established STH2. Moreover, multidose infusions of oxygenated esmolol cardioplegia main- 
tained complete protection for normothermic ischemic periods as long as 90 minutes. Esmolol cardioplegia thus may provide on efficacious alternative to hyperkalemia.

Dr Ryuzo Bessho was a visiting research fellow from the Division of Cardiovascular Surgery, the Department of Surgery II, Nippon Medical School, Tokyo, Japan.

\section{References}

1. Magee PG, Gardner TJ, Flaherty JT, Bulkley BH, Goldman RA, Gott VL. Improved myocardial protection with propranolol during induced ischemia. Circulation. 1980;62(2 Pt 2):I49-56.

2. Manning AS, Keogh JM, Coltart DJ, Hearse DJ. The effect of beta blockade and partial agonist activity during myocardial ischemia. $A d v$ Myocardiol. 1983;4:513-20.

3. Lu HR, Vandeplassche G, Wouters L, Flameng W, Borgers M. Effects of beta-adrenoceptor antagonists on cardiac function in ischemicreperfused myocardium of the isolated working rabbit heart. Eur J Pharmacol. 1990;184:65-74.

4. Khandoudi N, Percevault-Albadine J, Bril A. Comparative effects of carvedilol and metoprolol on cardiac ischemia-reperfusion injury. J Cardiovasc Pharmacol. 1998;32:443-51.

5. Kloner RA, Kirshenbaum J, Lange R, Antman EM, Braunwald E. Experimental and clinical observations on the efficacy of esmolol in myocardial ischemia. Am J Cardiol. 1985;56:40F-8F.

6. Mueller HS, Ayres SM. Propranolol decreases sympathetic nervous activity reflected by plasma catecholamines during evolution of myocardial infarction in man. J Clin Invest. 1980;65:338-46.

7. Opie LH, Owen P, Thomas M, Samson R. Coronary sinus lactate measurements in assessment of myocardial ischemia. Comparison with changes in lactate-pyruvate and beta-hydroxybutyrate-acetoacetate ratios and with release of hydrogen, phosphate and potassium ions from the heart. Am J Cardiol. 1973;32:295-305.

8. Nies AS, Shand DG. Clinical pharmacology of propranolol. Circulation. 1975;52:6-15.

9. Liu XK, Engelman RM, Agrawal HR, Das DK. Preservation of membrane phospholipids by propranolol, pindolol, and metoprolol: a novel mechanism of action of beta-blockers. J Mol Cell Cardiol. 1991;23:1091-100.

10. Erhardt PW, Woo CM, Gorczynski RJ, Anderson WG. Ultra-shortacting beta-adrenergic receptor blocking agents. 1. (Aryloxy)propanolamines containing esters in the nitrogen substituent. J Med Chem. $1982 ; 25: 1402-7$.

11. Erhardt PW, Woo CM, Anderson WG, Gorczynski RJ. Ultra-shortacting beta-adrenergic receptor blocking agents. 2. (Aryloxy)propanolamines containing esters on the aryl function. J Med Chem. 1982;25: 1408-12.

12. Quon CY, Stampfli HF. Biochemical properties of blood esmolol esterase. Drug Metab Dispos. 1985;13:420-4.

13. Gorczynski RJ. Basic pharmacology of esmolol. Am J Cardiol. 1985; $56: 3 \mathrm{~F}-13 \mathrm{~F}$.

14. Sweeney MS, Frazier OH. Device-supported myocardial revascularization: safe help for sick hearts. Ann Thorac Surg. 1992;54:1065-70.

15. Mehlhorn U, Sudkamp M. Beta-blockade as an alternative to cardioplegia. Thorac Cardiovasc Surg. 1998;46 Suppl 2:302-7.

16. Mehlhorn U, Sauer H, Kuhn-Regnier F, Sudkamp M, Dhein S, Eberhardt F, et al. Myocardial beta-blockade as an alternative to cardioplegic arrest during coronary artery surgery. Cardiovasc Surg. 1999;7: $549-57$.
17. Kuhn-Regnier F, Natour E, Dhein S, Dapunt O, Geissler HJ, LaRose $\mathrm{K}$, et al. Beta-blockade versus Buckberg blood-cardioplegia in coronary bypass operation. Eur J Cardiothorac Surg. 1999;15:67-74.

18. Pirk J, Kellovsky P. An alternative to cardioplegia. Ann Thorac Surg. 1995;60:464-5.

19. Fukata M, Konishi T, Higuchi K, Akishima S. [A case of CABG under the cardiac arrest induced by a short acting beta-blocker without clamping the aorta]. Nippon Kyobu Geka Gakkai Zasshi. 1997;45: 1591-5.

20. Bessho R, Chambers DJ. Myocardial protection: the efficacy of the ultra-short-acting beta-blocker, esmolol, as a cardioplegic agent. J Thorac Cardiovasc Surg. 2001;122:993-1003.

21. Bessho R, Chambers DJ. Experimental study of intermittent crossclamping with fibrillation and myocardial protection: reduced injury from shorter cumulative ischemia or intrinsic protective effect? $J$ Thorac Cardiovasc Surg. 2000;120:528-37.

22. Lucas SK, Elmer EB, Flaherty JT, Prodromos CC, Bulkley BH, Gott $\mathrm{BL}$, et al. Effect of multiple-dose potassium cardioplegia on myocardial ischemia, return of ventricular function, and ultrastructural preservation. J Thorac Cardiovasc Surg. 1980;80:102-10.

23. Bonchek LI, Burlingame MW, Vazales BE, Lundy EF, Gassmann CJ. Applicability of noncardioplegic coronary bypass to high-risk patients: selection of patients, technique, and clinical experience in 3000 patients. J Thorac Cardiovasc Surg. 1992;103:230-7.

24. Anderson JR, Hossein-Nia M, Kallis P, Pye M, Holt DW, Murday AJ, et al. Comparison of two strategies for myocardial management during coronary artery operations. Ann Thorac Surg. 1994;58:768-72.

25. Ledingham SJ, Braimbridge MV, Hearse DJ. Improved myocardial protection by oxygenation of St. Thomas' Hospital cardioplegic solutions: studies in the rat. J Thorac Cardiovasc Surg. 1988;95:103-11.

26. von Oppell UO, King LM, Du Toit EF, Owen P, Reichart B, Opie LH. Effect of oxygenation and consequent $\mathrm{pH}$ changes on the efficacy of St. Thomas' Hospital cardioplegic solution. J Thorac Cardiovasc Surg. 1991;102:396-404.

27. Chambers DJ, Kosker S, Takahashi A, Sakai A, Baharakakis S, Manzanera G, et al. Comparison of standard (non-oxygenated) vs. oxygenated St. Thomas' Hospital cardioplegic solution No. 2 (Plegisol). Eur J Cardiothorac Surg. 1990;4:549-55.

28. Lochner A, Lloyd L, Brits W, Coetzee A. Oxygenation of cardioplegic solutions: a note of caution. Ann Thorac Surg. 1991;51:777-87.

29. Bernard M, Menasché P, Canioni P, Fontanarava E, Grousset C, Piwnica A, et al. Influence of the $\mathrm{pH}$ of cardioplegic solutions on intracellular $\mathrm{pH}$, high-energy phosphates, and postarrest performance: protective effects of acidotic, glutamate-containing cardioplegic perfusates. J Thorac Cardiovasc Surg. 1985;90:235-42.

30. Baker JE, Boerboom LE, Olinger GN. Age and protection of the ischemic myocardium: is alkaline cardioplegia appropriate? Ann Thorac Surg. 1993;55:747-55.

31. Rahn H, Reeves RB, Howell BJ. Hydrogen ion regulation, temperature, and evolution. Am Rev Respir Dis. 1975;112:165-72.

32. Toleikis PM, Tomlinson CW. Myocardial functional preservation during ischemia: influence of beta blocking agents. Mol Cell Biochem. 1997;176:205-10.

33. Murthy VS, Frishman WH. Controlled beta-receptor blockade with esmolol and flestolol. Pharmacotherapy. 1988;8:168-82.

34. Wollenberger A, Krause EG. Metabolic control characteristics of the acutely ischemic myocardium. Am J Cardiol. 1968;22:349-59.

35. Vatner SF, Baig H, Manders WT, Ochs H, Pagani M. Effects of propranolol on regional myocardial function, electrograms, and blood flow in conscious dogs with myocardial ischemia. J Clin Invest. 1977;60:353-60.

36. Gross GJ, Winbury MM. Beta adrenergic blockade on intramyocardial distribution of coronary blood flow. J Pharmacol Exp Ther. 1973;187: 451-64. 